\title{
Excesso de peso entre jovens de um município do semiárido brasileiro: estudo de base populacional
}

\author{
Overweight among young people in a city \\ in the Brazilian semiarid region: a population-based study
}

\begin{abstract}
Aline Rodrigues Monteiro ${ }^{1}$ Samuel Carvalho Dumith ${ }^{1,2}$ Tatiane Santos Gonçalves ${ }^{3}$ Juraci Almeida Cesar ${ }^{2}$
\end{abstract}

${ }^{1}$ Programa de PósGraduação em Ciências da Saúde, Universidade Federal do Rio Grande (FURG). R. General Osório s/n, Centro, CEP 96203-900 Rio Grande RS Brasil.

lihhmonteiro@gmail.com ${ }^{2}$ Programa de Pós-

Graduação em Saúde Pública, Faculdade de Medicina, FURG. Rio Grande RS Brasil.

${ }^{3}$ Secretaria de Assistência Social, Prefeitura Municipal de Paulo Afonso. Paulo Afonso BA Brasil.
Abstract The scope of this article is to measure the prevalence and identify factors associated with excess weight among young people living in the city of Caracol, Piaui, Brazil. Qualified interviewers applied a standardized questionnaire and assessed the anthropometric measurements of all young people (13 to 19 years of age) in their households. The questionnaires sought information on demographic, socioeconomic and behavioral characteristics. The outcome was overweight in young people (BMI > $1 z$ score) calculated on the basis of body mass index (BMI) and classified in accordance with the new parameters of the World Health Organization. Poisson regression with robust adjustment of variance was used for the statistical analysis. Of the 1,088 young people studied, $10.5 \%$ were overweight. The occurrence of the outcome varied from 6\% for young people belonging to the second income quartile to $19 \%$ for adolescents with a height deficit for their age. Even with the prevalence of overweight being below the national average in the semiarid region, the factors associated with their occurrence are already equal to the other areas of Brazil and there is a pressing need for early intervention aimed at preventing and reducing the current overweight levels.

Key words Overweight, Obesity, Adolescent, Associated factors
Resumo O objetivo deste artigo é medir a prevalência e identificar fatores associados ao excesso de peso entre jovens residentes no município de Caracol - PI. Através de questionário padronizado e medidas antropométricas de todos os jovens, nos domicílios. Investigaram-se características demográficas, socioeconômicas e comportamentais. $O$ desfecho foi o excesso de peso nos jovens (IMC > 1 escore z), calculado a partir do indice de massa corpórea (IMC) e classificado conforme as novas curvas da Organização Mundial da Saúde. Na análise estatística, utilizou-se regressão de Poisson com ajuste robusto de variância. Dos 1088 jovens estudados, 10,5\% apresentaram excesso de peso. A ocorrência do desfecho variou de 6\% para os jovens pertencentes ao segundo quartil de renda a 19\% para os jovens com déficit de altura para idade. Mesmo com a prevalência de excesso de peso ainda se mostrando abaixo da média nacional no semiárido, os fatores associados a sua ocorrência já se igualam aos das demais regiões do Brasil e parece evidente a necessidade de intervenção precoce visando à prevenção e à redução desses níveis atuais.

Palavras-chave Sobrepeso, Obesidade, Jovens, Fatores associados 


\section{Introdução}

Nas últimas quatro décadas, como reflexo da transição nutricional, houve aumento significativo do excesso de peso, que é caracterizado pela prevalência de sobrepeso ligada à de obesidade. Devido a esse aumento, a obesidade passou a ser encarada não só como uma doença crônica, mas como uma epidemia global ${ }^{1}$. O aumento do excesso de peso consolidou-se em virtude do crescimento da dieta rica em gorduras, açúcares, alimentos refinados e da redução em carboidratos complexos e fibras, juntamente com a diminuição da atividade física ${ }^{2}$, sendo observado especialmente durante a infância e a adolescência ${ }^{1}$.

Os determinantes do excesso de peso, principalmente na faixa infantojuvenil, consistem em um conjunto de fatores biológicos, comportamentais e ambientais. Hábitos alimentares, tempo que os jovens permanecem assistindo à televisão, sobrepeso e obesidade dos pais e peso ao nascer são alguns dos principais determinantes do excesso de peso nesta faixa etária, juntamente com a falta de atividade física ${ }^{2,3}$.

Destaca-se que a associação de elevadas prevalências de excesso de peso aos fatores externos e/ ou comportamentais é de aproximadamente 95\%, sendo apenas $5 \%$ dos casos associados a fatores genéticos ou neuroendócrinos nesta faixa etária ${ }^{3}$.

É sabido que a obesidade é fator de risco para diversas outras doenças crônicas não transmissíveis (DCNT), tais como: doença coronariana, hipertensão, diabetes, dislipidemias, entre outras ${ }^{4}$. Em razão disso, torna-se importante estudar os estágios mais precoces de seu desenvolvimento, dentre os quais a adolescência, visto a existência de estudos que comprovam a associação da obesidade na fase infantojuvenil com as DCNT na idade adulta ${ }^{5}$.

Os Estados Unidos apresentam a maior prevalência de jovens acima do peso, em média $35 \%{ }^{6}$. No Brasil, estudos recentes de base populacional mostram que essa prevalência nos jovens brasileiros está em torno de $25 \%{ }^{7}$. Segundo a Pesquisa Nacional de Saúde do Escolar - PENSE de $2009^{8}$, a região Nordeste apresenta as menores prevalências de excesso de peso entre jovens, com cerca de $16 \%$. Isso sugere que esta região ainda está em fase de transição nutricional, situação em que o problema da desnutrição vem dando lugar à obesidade, e as prevalências de excesso de peso, em constante aumento, tornam-se cada vez mais preocupantes nesta região.

Tendo em vista que a intervenção com relação a mudanças no estilo de vida, tais como há- bitos alimentares e atividade física, é mais efetiva quanto mais precoce e que a chance de um indivíduo apresentar obesidade na idade adulta é maior para aqueles que apresentam excesso de peso aos 18 anos de idade do que para um jovem com peso normal ${ }^{9}$, é fundamental que se conheça a magnitude do sobrepeso e da obesidade e seus determinantes na faixa etária infantojuvenil.

A fim de colaborar com as estratégias de prevenção para auxiliar nas políticas públicas no combate ao excesso de peso, o objetivo do presente estudo foi avaliar a prevalência de excesso de peso (sobrepeso e obesidade) e fatores associados, entre jovens do município de Caracol Piauí.

\section{Metodologia}

Trata-se de um estudo epidemiológico de delineamento transversal desenvolvido como parte de um projeto mais amplo cujo objetivo era reduzir a ocorrência de gravidez na adolescência e estudar questões relacionadas ao consumo de drogas entre jovens residentes no município de Caracol, PI. Localizado no sul do Estado, Caracol fica a aproximadamente $600 \mathrm{~km}$ de Teresina, a capital.

Os dados utilizados neste estudo foram coletados no período de janeiro e fevereiro de 2011. $\mathrm{Na}$ ocasião, Caracol possuía pouco mais de 10 mil habitantes. Foram incluídos no estudo todos os jovens com idades entre 13 e 19 anos residentes nas áreas urbana e rural do município. Partiuse dessa faixa etária em razão de o estudo piloto do projeto original mostrar que aproximadamente $90 \%$ dos jovens com idades entre 10 e 12 anos não se mostraram capazes de ler, entender e responder ao questionário autoaplicável. Foram excluídas das análises as jovens que estavam grávidas no momento da entrevista.

Este trabalho incluiu 1112 jovens previamente entrevistados através de dois questionários padronizados aplicados no domićlío do participante. O primeiro, aplicado pelo entrevistador, com questões mais abrangentes, investigou informações referentes à família e ao adolescente, tais como características demográficas (cor da pele, idade, sexo), condições socioeconômicas (escolaridade, renda familiar) e comportamentais (nível de atividade física, frequência de educação física na escola e horas que assiste TV por dia). $\mathrm{O}$ segundo questionário era confidencial e autoaplicável. A sua aplicação ocorria da seguinte maneira: o material era entregue ao adolescente em um envelope fechado, por ele aberto, lido, res- 
pondido, depois colocado de volta no envelope, lacrado e devolvido ao entrevistador. Este questionário buscava informações sobre uso de drogas lícitas e ilícitas, entre outras variáveis, como início da vida sexual, ocorrência prévia de gravidez e de aborto. As medidas antropométricas dos jovens foram coletadas pelos entrevistadores, no domicílio, através de balança digital portátil com precisão de $100 \mathrm{~g}$ e para até 150 quilos e antropômetro Alturaexata com precisão de 1 centímetro.

Para realização da coleta de dados deste estudo, os entrevistadores, um total de 14 estudantes de graduação do Campus de São Raimundo Nonato, da Universidade Estadual do Piauí (UESPI), foram selecionados e treinados durante quatro dias. Destes, apenas oito participaram do estudo. $\mathrm{O}$ treinamento foi realizado a partir da leitura dos questionários e do manual de instruções e simulações de entrevistas.

$\mathrm{O}$ estudo piloto foi realizado no quinto dia em São Raimundo Nonato e visava testar cada questão. Os entrevistadores foram divididos em duplas e designados a áreas distintas do município, anteriormente mapeadas e numeradas. Já o controle de qualidade foi realizado com a revisão dos questionários e repetição parcial de 5\% das entrevistas a fim de confirmar a realização das mesmas e comparar as respostas obtidas. Todos os questionários foram codificados e revisados pelos entrevistadores e duplamente digitados em ordem inversa, por diferentes digitadores usando o programa Epi-Info $6.0^{10}$.

As variáveis independentes avaliadas foram: sexo (masculino e feminino), idade em anos completos (categorias 13-14, 15-16 e 17-19) e cor da pele autorreferida (branca, parda e preta), escolaridade em anos completos com aprovação $(1-4,5-8$ e $\geq 9)$ renda familiar (em reais, dividida em quartis), frequência de atividade física (mais de $1 \mathrm{~h} /$ dia de duração, menos de $1 \mathrm{~h} /$ dia de duração), realização de educação física na escola (em dias por semana) e tempo que assiste à TV por dia (em horas e minutos por dia). A partir das variáveis peso e altura, foi estudada a variável déficit de altura para idade (A/I) sendo considerado déficit quando $\mathrm{A} / \mathrm{I}<-2$ escore z. O desfecho foi obtido através das variáveis antropométricas de peso e altura, determinando o excesso de peso por meio do índice de massa corporal $(\mathrm{IMC}=$ peso/altura ${ }^{2}$ ). Os jovens foram classificados de acordo com as curvas da OMS $(2007)^{11}$ e considerou-se com excesso de peso os jovens que apresentaram IMC $>1$ escore $z$.

A análise bruta constou da obtenção de listagem de frequências, enquanto a ajustada obede- ceu ao modelo hierárquico previamente definido em três níveis. No primeiro, mais distal, foram incluídas as variáveis demográficas e socioeconômicas dos jovens; no segundo, as comportamentais e no terceiro, o mais proximal, a variável biológica altura para idade. Para a análise estatística bruta e ajustada utilizou-se o programa Stata versão $11.2^{12}$. As associações entre as variáveis independentes e o excesso de peso foram feitas por meio de Regressão de Poisson simples e multivariável, com ajuste robusto para variância, e o nível de significância estatística adotada para as análises foi de 5\%. Mantiveram-se no modelo ajustado as variáveis com valor p menor que 0,20.

Os jovens maiores de 18 anos assinaram o Termo de Consentimento Livre e Esclarecido. Para os demais, o termo era assinado pelo seu responsável. O projeto foi aprovado pelo Comitê de Ética em Pesquisa da Faculdade de Medicina da Universidade Federal de Pelotas (CEPAS/UFPel) e financiado pelo Conselho Nacional de Desenvolvimento Científico e Tecnológico (CNPq).

\section{Resultados}

Dos 1.133 jovens estudados, houve 21 perdas, sendo 12 entre os rapazes e 9 entre as moças, totalizando 98,1\% dos indivíduos elegíveis. Considerando-se que foram excluídas do nosso estudo 24 jovens que estavam grávidas no momento da coleta de dados, a amostra final ficou composta por 1088 sujeitos. A idade variou entre 13 e 19 anos (média $=15,7 ; \mathrm{DP}=1,9) ; 53 \%$ eram do sexo feminino, $83 \%$ eram de cor parda, $53 \%$ estavam entre a $5^{a}$ e $8^{a}$ série do ensino fundamental e $33 \%$ não possuíam renda familiar mensal. A mediana de renda familiar foi de $\mathrm{R} \$ 280,00$ e a mediana per capita ficou em $\mathrm{R} \$ 66,00$ e $65 \%$ da amostra recebia o auxílio Bolsa Família do Governo Federal. Em relação aos hábitos comportamentais, 56\% assistiam mais de 2 horas de televisão (TV) por dia, $43 \%$ não tinham aula de educação física na escola e $80 \%$ eram insuficientemente ativos pelo critério de uma hora de atividade física/dia. Destaca-se que $5 \%$ dos jovens possuíam baixa altura para sua idade.

A prevalência de excesso de peso dos jovens foi de 10,5\% (IC95\% 8,7 a 11,3). A Tabela 1 apresenta a prevalência de excesso de peso conforme as variáveis socioeconômicas, demográficas e comportamentais analisadas. Observa-se que a ocorrência do desfecho variou de $6,0 \%$ para os jovens pertencentes ao segundo quartil de renda a 19,3\% para os jovens com déficit de altura para idade. 
Os grupos mais propensos a apresentar excesso de peso foram os jovens de cor de pele branca, aqueles que assistiam menos de 2 horas/dia de televisão e os indivíduos com baixa estatura para a idade, tanto na análise bruta quanto na ajustada (Tabela 2). As demais variáveis analisadas não apresentaram associação estatisticamente significativa com o desfecho em estudo. Importante salientar que a prevalência de excesso de peso apresentou uma associação em formato de " $U$ " com a renda familiar, ou seja, mostrou-se mais elevada nos grupos de maior e de menor renda.

Tabela 1. Prevalência de excesso de peso de acordo com as características demográficas, socioeconômicas e comportamentais dos jovens residentes no município de Caracol, Piauí, Brasil, 2011.

\begin{tabular}{|c|c|c|}
\hline Variável & $\mathbf{N}$ & $\begin{array}{l}\text { Prevalência de } \\
\text { excesso de peso }\end{array}$ \\
\hline \multicolumn{3}{|l|}{ Sexo } \\
\hline Masculino & 519 & $9,5 \%$ \\
\hline Feminino & 569 & $11,4 \%$ \\
\hline \multicolumn{3}{|l|}{ Idade (anos) } \\
\hline $13-14$ & 340 & $10,3 \%$ \\
\hline $15-16$ & 372 & $11,8 \%$ \\
\hline $17-19$ & 376 & $9,3 \%$ \\
\hline \multicolumn{3}{|l|}{ Cor da pele } \\
\hline Branca & 143 & $18,2 \%$ \\
\hline Parda & 895 & $9,4 \%$ \\
\hline Preta & 50 & $8,0 \%$ \\
\hline \multicolumn{3}{|l|}{ Escolaridade (anos) } \\
\hline $1-4$ & 198 & $9,6 \%$ \\
\hline $5-8$ & 613 & $11,9 \%$ \\
\hline$\geq 9$ & 277 & $7,9 \%$ \\
\hline \multicolumn{3}{|l|}{ Renda (quartis) } \\
\hline 1 (menor) & 359 & $12,0 \%$ \\
\hline 2 & 183 & $6,0 \%$ \\
\hline 3 & 275 & $9,1 \%$ \\
\hline 4 & 271 & $12,9 \%$ \\
\hline \multicolumn{3}{|l|}{ TV (horas/dia) } \\
\hline$\leq 2$ & 474 & $14,0 \%$ \\
\hline$>2$ & 614 & $7,8 \%$ \\
\hline \multicolumn{3}{|c|}{ Aulas de Educação Física (nº) } \\
\hline 0 & 454 & $11,9 \%$ \\
\hline 1 & 202 & $7,4 \%$ \\
\hline 2 & 314 & $10,5 \%$ \\
\hline 3 & 118 & $10,2 \%$ \\
\hline \multicolumn{3}{|c|}{ Ativo (> 300min/semana) } \\
\hline Não & 859 & $10,3 \%$ \\
\hline $\operatorname{Sim}$ & 229 & $11,4 \%$ \\
\hline \multicolumn{3}{|l|}{ Déficit de altura } \\
\hline Não & 1030 & $10,0 \%$ \\
\hline Sim & 57 & $19,3 \%$ \\
\hline Total & 1088 & $10,5 \%$ \\
\hline
\end{tabular}

\section{Discussão}

Como ponto forte da pesquisa destaca-se o fato de incluir todos os adolescentes de um município em uma região em que avaliações deste tipo são extremamente raras, o que torna importante o conhecimento atual da saúde nutricional dos adolescentes dessa região.

Dentre as limitações da pesquisa, pode-se incluir o tipo de delineamento utilizado, do tipo transversal, sendo o desfecho e as exposições coletadas em um mesmo momento, em que os achados podem ser afetados pelo viés de causalidade reversa, ou seja, alguns dos fatores associados investigados, como os comportamentais de nível de atividade física e horas de TV/dia podem ser influenciados pela ocorrência do desfecho. Por isso, as associações identificadas não devem ser interpretadas como relações causais.

Com relação aos resultados encontrados, a prevalência de excesso de peso da amostra $(10,5 \%)$ está abaixo da média nacional $(25 \%)^{7}$ e abaixo do esperado em uma população de referência. A frequência esperada numa curva de distribuição normal, considerando-se como ponto de corte escore $z>1$, é de $16 \%$ de jovens acima do peso. Isso se deve à região Nordeste estar em fase de transição nutricional, na qual os índices de baixo peso ainda se encontram relativamente altos quando comparados a outras regiões. Quando esses achados são comparados a outros estudos mais recentes realizados no Norte e Nordeste do Brasil, nota-se uma grande variação da prevalência de excesso de peso, de $8,4 \%{ }^{13}$ a $26 \%{ }^{14}$. Porém, devido à escassez de estudos nesta região e às diferentes formas metodológicas aplicadas, torna-se difícil a comparação de prevalências.

$\mathrm{O}$ estudo mais atual realizado no interior do Piauí por Moura et al. ${ }^{15}$, em 2012, encontrou uma prevalência de $15,8 \%$ de excesso de peso em jovens estudados nesta mesma faixa etária. A histórica desigualdade no processo de desenvolvimento socioeconômico, em especial na Região Nordeste do país, e o estilo de vida mais dificultado parece ainda justificar as diferenças percentuais do excesso de peso com relação ao que ocorre nas demais regiões do Brasil. Segundo a POF de $2010^{7}$, os maiores índices de excesso de peso estão nas regiões Sudeste $(39,7 \%)$, Centro-Oeste $(37,9 \%)$ e Sul $(36,3 \%)$.

Ainda assim, a prevalência de excesso de peso vem aumentando na região Nordeste, onde as proporções variam de $4 \%$ em um estudo em Teixeira de Freitas, Bahia ${ }^{16}$, feito em 2001, a 26,0\% no município de Queimados na Paraíba, em 
Tabela 2. Análise bruta e ajustada para os fatores associados ao excesso de peso dos jovens residentes no município de Caracol, Piauí, Brasil, 2011 ( $\mathrm{N}=1088)$.

\begin{tabular}{|c|c|c|c|c|c|}
\hline Nível de análise ${ }^{*}$ & Variável & $\begin{array}{r}\text { Análise Bruta } \\
\text { RP (IC95\%) }\end{array}$ & $\begin{array}{c}\text { Valor } \\
\text { p }\end{array}$ & $\begin{array}{r}\text { Análise Ajustada } \\
\text { RP (IC95\%) }\end{array}$ & $\begin{array}{c}\text { Valor } \\
\text { p }\end{array}$ \\
\hline \multirow[t]{3}{*}{ I (Distal) } & Sexo & & 0,29 & & 0,18 \\
\hline & Feminino & $1,21(0,85-1,72)$ & & $1,27(0,89-1,82)$ & \\
\hline & Masculino & 1,00 & & 1,00 & \\
\hline \multirow[t]{4}{*}{ I (Distal) } & Idade (anos) & & 0,53 & & 0,43 \\
\hline & $13-14$ & 1,00 & & 1,00 & \\
\hline & $15-16$ & $1,10(0,71-1,72)$ & & $1,03(0,66-1,61)$ & \\
\hline & $17-19$ & $1,27(0,83-1,93)$ & & $1,29(0,84-1,97)$ & \\
\hline \multirow[t]{4}{*}{ I (Distal) } & Cor da pele & & 0,01 & & 0,01 \\
\hline & Branca & $1,94(1,29-2,90)$ & & $1,82(1,21-2,72)$ & \\
\hline & Parda & 1,00 & & 1,00 & \\
\hline & Preto & $0,85(0,33-2,23)$ & & $0,88(0,33-2,35)$ & \\
\hline \multirow[t]{4}{*}{ I (Distal) } & Escolaridade (anos) & & 0,19 & & 0,10 \\
\hline & $1-4$ & 1,00 & & 1,00 & \\
\hline & $5-8$ & $1,24(0,77-2,01)$ & & $1,17(0,73-1,89)$ & \\
\hline & $\geq 9$ & $0,83(0,46-1,49)$ & & $0,71(0,39-1,27)$ & \\
\hline \multirow[t]{5}{*}{ I (Distal) } & Renda (quartis) & & 0,09 & & 0,06 \\
\hline & 1 (menor) & 1,00 & & 1,00 & \\
\hline & 2 & $0,50(0,27-0,95)$ & & $0,49(0,26-0,93)$ & \\
\hline & 3 & $0,76(0,48-1,22)$ & & $0,75(0,47-1,19)$ & \\
\hline & 4 & $1,08(0,71-1,64)$ & & $1,11(0,73-1,70)$ & \\
\hline II & TV/dia (horas) & & $<\mathbf{0 , 0 1}$ & & $<\mathbf{0 , 0 1}$ \\
\hline \multirow[t]{2}{*}{ (Intermediário) } & $\leq 2 \mathrm{~h} / \mathrm{dia}$ & 1,00 & & 1,00 & \\
\hline & $>2 \mathrm{~h} / \mathrm{dia}$ & $0,56(0,39-0,80)$ & & $0,57(0,40-0,80)$ & \\
\hline \multirow[t]{5}{*}{$\begin{array}{c}\text { II } \\
\text { (Intermediário) }\end{array}$} & $\begin{array}{l}\text { Aulas de Educação Física } \\
\text { (vezes/semana) }\end{array}$ & & 0,40 & & 0,45 \\
\hline & 0 & $1,17(0,65-2,12)$ & & $1,21(0,67-2,15)$ & \\
\hline & 1 & $0,73(0,35-1,51)$ & & $0,77(0,38-1,55)$ & \\
\hline & 2 & $1,03(0,55-1,93)$ & & $1,01(0,55-1,85)$ & \\
\hline & 3 & 1,00 & & 1,00 & \\
\hline \multirow[t]{3}{*}{ II (Intermediário) } & Ativo (>300min/semana) & & 0,63 & & 0,50 \\
\hline & Não & $0,90(0,60-1,37)$ & & $0,86(0,57-1,32)$ & \\
\hline & Sim & 1,00 & & 1,00 & \\
\hline \multirow[t]{3}{*}{ III (Proximal) } & $\begin{array}{l}\text { Déficit de altura } \mathrm{p} / \text { idade } \\
\text { (escore } \mathrm{z}<-2 \mathrm{DP} \text { ) }\end{array}$ & & 0,02 & & 0,04 \\
\hline & Não & 1,00 & & 1,00 & \\
\hline & Sim & $1,93(1,10-3,39)$ & & $1,85(1,03-3,31)$ & \\
\hline
\end{tabular}

* Cada variável foi ajustada para aquelas do mesmo nível e de níveis acima de acordo com o modelo hierárquico e foram mantidas no modelo final aquelas com valor $\mathrm{P}<0.20$.

$2013^{14}$. Caracterizando, assim, a transição nutricional que sofre ainda esta região, onde a desnutrição, hoje, vem dando lugar ao excesso de peso.

$\mathrm{Na}$ amostra do presente estudo, encontrouse $5 \%$ (dados não apresentados) de baixo peso, utilizando como ponto de corte $<-2$ escore $\mathrm{z}$ de acordo com o critério da $\mathrm{OMS}^{11}$. Nota-se que nesta região predomina ainda o baixo peso quando comparada com as demais regiões do país, onde a média de desnutrição fica em torno de $2 \%{ }^{7}$. A prevalência de obesidade, utilizando como pon- to de corte escore $\mathrm{z}>2$, foi de $1,8 \%$ (dados não apresentados) estando abaixo do esperado para uma população de referência e menor do que as demais regiões do país (em torno de $5 \%{ }^{7}$ ). Ou seja, a região Nordeste encontra-se em fase de transição nutricional, em que as prevalências de baixo peso ainda encontram-se elevadas e as de obesidade estão abaixo da média nacional.

De acordo com as variáveis estudadas, no presente estudo não foram encontradas diferenças significativas nas prevalências de excesso de 
peso entre os sexos, similarmente ao que foi encontrado no estudo de Ogden et al. ${ }^{17}$, em 2002. Já os resultados da Pesquisa Nacional de Saúde Escolar (Pense 2009) ${ }^{8}$ mostram que as prevalências de excesso de peso em adolescentes de 13-15 anos, segundo o critério definido pela $\mathrm{OMS}^{11}$, foram de aproximadamente $21 \%$ entre as meninas e de $19 \%$ entre os meninos.

Embora a prevalência de excesso de peso tenha sido maior nos adolescentes de 15-16 anos, esta diferença não foi estatisticamente significativa. No estudo de Moura et al. ${ }^{15}$, de 2012, com adolescentes residentes no estado do Piauí, de idades entre 11 a 19 anos, as prevalências de excesso de peso também foram maiores naqueles com idade acima de 15 anos, sendo mais elevada no sexo feminino.

O presente estudo apontou para maior risco de excesso de peso em jovens de cor de pele branca, sendo essa associação estatisticamente significativa. Pode-se inferir a essa associação os fatores ambientais como os principais predisponentes, e a literatura sugere que este aumento da prevalência para o grupo étnico branco sofre efeito das diferenças socioeconômicas e estilo de vida entre os grupos ${ }^{18}$. Estudos mostram que o excesso de peso é maior em pessoas de cor da pele branca e do sexo masculino, já outros não encontraram diferenças significativas entre a cor da pele ${ }^{19-21}$.

A literatura mostra que em regiões ou estados menos desenvolvidos, a proporção de excesso de peso cresce conforme o aumento da renda ${ }^{22,23}$. Importante ressaltar que os jovens estudados são de uma região pobre do Nordeste, sendo boa parte de baixa renda, o que torna difícil estabelecer associações com o nível socioeconômico, podendo assim ter afetado nos resultados e nas poucas associações encontradas. O presente estudo mostrou uma associação em formato de "U", na qual os jovens mais propensos ao excesso de peso encontram-se nos níveis de menor e de maior renda, caracterizando a realidade de transição nutricional que ainda sofre o nordeste brasileiro. Dados da POF 2008-20097 confirmam a associação do aumento da prevalência de excesso de peso conforme a renda, porém, também mostra que, ao longo do tempo, a prevalência de sobrepeso e obesidade na população com baixa renda tem aumentado.

É fato que em países de baixa renda a transição nutricional é marcada por rápido crescimento das taxas de sobrepeso e obesidade em grupos de alto nível socioeconômico. Porém, com o tempo, esse quadro inverte-se e mostra-se próximo do observado nos países de alta renda, com redução da prevalência de obesidade nas classes mais favorecidas. Tal fato é explicado por alguns estudos pelo maior acesso a informação sobre padrões dietéticos e hábitos de vida mais saudáveis pelos grupos de renda mais alta ${ }^{24-26}$ Acredita-se que o Brasil está nesse mesmo caminho, onde nas regiões Sul e Sudeste esta prevalência já se mostra mais elevada nos indivíduos mais pobres, mas na região Nordeste ainda está mais alta nas pessoas de maior renda, porém já nota-se um declínio nesta diferença ${ }^{27}$. De acordo com a análise feita pelo Instituto de Pesquisa Econômica Aplicada dos resultados da Pesquisa Nacional por Amostra de Domicílios, PNAD - 2008 $8^{28}$, há importantes dados relativos à queda da desigualdade de renda no Brasil, incluindo principalmente a região Nordeste, mostrando o aumento do acesso a bens entre as famílias com renda familiar per capita inferior a meio salário mínimo, ou seja, a pobreza atual está caracterizada por mais acesso ao consumo de bens e serviços, o que inclui também a alimentação.

Em relação aos aspectos comportamentais estudados na amostra, é sabido que a relação dos jovens com a televisão é evidenciada em muitos estudos $^{19,29}$, estando altamente e significativamente associada ao excesso de peso. Porém os achados da presente pesquisa mostram essa relação inversa, na qual quem assiste menos horas de TV/dia tem maior prevalência de excesso de peso. Em uma revisão sistemática feita por Rossi e Vasconcelos $^{30}$, em 2010, 60\% dos estudos mostraram associação positiva entre o tempo despendido em frente à televisão e o excesso de peso nas crianças e adolescentes, porém em um dos estudos, de Waller et al. ${ }^{31}$, em 2003, os resultados foram similares ao do presente trabalho, em que essa relação inversa foi explicada pela baixa prevalência de excesso de peso encontrada nos jovens estudados $(9,1 \%)$, comparado com outros estudos. E mostrou também que esses jovens assistiam durante pouco tempo à televisão, concluindo que $\mathrm{o}$ fato de permanecer em frente à TV, portanto, não é fator associado ao sobrepeso, já que os jovens eutróficos assistiam significativamente mais à TV do que os jovens com excesso de peso.

Já em relação à atividade física, a presente pesquisa não mostrou associação entre inatividade física e excesso de peso. Apesar de estar bem estabelecida em vários estudos essa relação, os resultados, entretanto, diferem de um trabalho para outro. Investigações de base populacional, como a de Silva et al. ${ }^{26}$, de 2008, não têm encontrado essa associação em seus resultados, enquanto estudos de base escolar como o de Souza et al..$^{32}$, em 2010, apontam para este dado. Este achado 
é difícil de ser explicado, pois não foi avaliado, no presente estudo, o consumo alimentar dos jovens, o que também pode estar interferindo nos resultados encontrados.

Sobre o déficit de altura para a idade, esta variável mostrou-se associada significativamente ao excesso de peso no presente estudo, apontando uma prevalência duas vezes maior nesses jovens. Este indicador, altura para idade, mede o crescimento linear e seu déficit relaciona-se a alterações cumulativas de longo prazo na situação nutricional e de saúde em geral. No Brasil, a estimativa mais recente da prevalência de baixa estatura em adolescentes é de 3,7\% nos meninos e $3 \%$ nas meninas 7 . Maior acúmulo de gordura subcutânea e maior prevalência de excesso de peso também já foram associados à baixa estatura em diversos estudos com jovens, como os realizados por Benefice et al. ${ }^{33}$, em 2001, e Florêncio et al..$^{34}$, em 2003. No Brasil, poucos são os trabalhos que avaliam a associação do déficit de estatura com o excesso de peso nessa faixa etária. Embora a presente pesquisa não tenha o objetivo de avaliar a composição corporal dos jovens, é possível que o elevado IMC/I observado entre aqueles com déficit de altura também venha acompanhado pelo incremento de tecido adiposo, o que pode ter influenciado na associação encontrada, em que os adolescentes mais baixos apresentaram maior propensão ao excesso de peso. Esse achado sugere a continuação do agravo de déficit nutricional que, provavelmente, determinou déficit de crescimento linear durante a infância nessa população.

Considerando os resultados deste e de outros estudos, torna-se evidente a necessidade de intervenções visando à prevenção e redução dos atuais níveis de excesso de peso. Também é importante que se crie estratégias para reduzir a desnutrição ainda presente neste município, e a realização de novos estudos para monitoramento destes dados. Além de ações individuais, é fundamental que as Políticas de Alimentação e Nutrição disponibilizem cuidados nas várias esferas em que os jovens se inserem. Nas escolas, principalmente, atenção à qualidade da merenda escolar e dos alimentos oferecidos nas cantinas, além da educação nutricional e da promoção à atividade física devem ser os focos das medidas preventivas e de tratamento para proporcionar uma melhor qualidade de vida na adolescência e, consequentemente, na vida adulta.

Conclui-se que a prevalência de excesso de peso encontrada na presente investigação foi mais baixa do que o esperado, porém já se torna preocupante por se tratar de um município com baixo IDH, onde a prevalência de excesso de peso vem crescendo apesar de as de baixo peso ainda se manterem elevadas. Espera-se com este estudo alertar sobre a necessidade de políticas públicas para o combate à obesidade também nessas áreas mais pobres da região Nordeste.

\section{Colaboradores}

AR Monteiro trabalhou na concepção e redação final do artigo; SC Dumith analisou os dados e revisou criticamente o artigo; TS Gonçalves supervisionou o trabalho de campo, a digitação dos dados e revisou o artigo; JA Cesar coordenou o trabalho de campo e revisou criticamente o artigo. Todos os autores leram e aprovaram a versão final enviada.

\section{Referências}

1. World Health Organization (WHO). Obesity: preventing and managing the global epidemic. Geneva: WHO; 2012. Report of a WHO consultation

2. Tardido AP, Falcão MC. O impacto da modernização na transição nutricional e obesidade. Rev Nutr 2006; 21(2):117-124

3. Carvalho EAA, Simão MTJ, Fonseca MC, Andrade RG, Ferreira MSG, Silva AF, Souza IPR, Fernandes BS. Obesidade: Aspectos epidemiológicos e prevenção. Rev Med de Minas Gerais 2013; 23(1):2238-3182

4. World Health Organization (WHO). Global health risks: mortality and burden of disease attributable to selected major risks. Geneva: WHO; 2009.

5. Torres KP, Torres SG, Guerra RO. Riscos cardiovasculares em adolescentes com diferentes graus de obesidade. Arq Bras Cardiol 2011; 96(3):205-211.

6. Health Behaviour in School-aged Children (HBSC). A World Health Organization cross-national study. St Andrews: University of St Andrews; 2009. 
7. Instituto Brasileiro de Geografia e Estatística (IBGE). Pesquisa de orçamentos familiares 2008-2009: antropometria e estado nutricional de crianças, adolescentes e adultos no Brasil. Rio de Janeiro: IBGE; 2010.

8. Instituto Brasileiro de Geografia e Estatística (IBGE). Pesquisa Nacional de Saúde do Escolar (Pense). Rio de Janeiro: IBGE; 2009.

9. Guo SS, Chumlea WC. Tracking of body mass index in children in relation to overweight in adulthood. Am J Clin Nutr 1999; 70(Supl.):145S-148.

10. Dean AG, Dean JA, Coulombier D, Brendel KA, Smith DC, Burton AH, Brendel KA, Smith DC, Dicker RC, Sullivan KM, Fagan RF. Epi-Info, Version 6: A Word Processing, Database, and Statistics Program for Epidemiology on Microcomputers. Atlanta: Centers of Disease Control and Prevention; 2002.

11. De Onis M, Onyango AW, Borghi E, Siyama, Nishida C, Siekmann J. Development of a WHO growth reference for school-aged children and adolescents. Bulletin of the World Health Organization 2007; 85(9):660-667.

12. StataCorp. Stata statistical software: release 11.2. College Station: Stata Corporation; 2011.

13. Abrantes MM, Lamounier JA, Colosimo EA. Prevalência de sobrepeso e obesidade em crianças e adolescentes das Regiões Sudeste e Nordeste do Brasil. J Pediatr 2002; 78(4):335-340.

14. Oliveira MAT, Nascimento MAM, Maia DF, Farias ALP. Prevalência de sobrepeso, obesidade e risco cardíaco de alunos do ensino médio. Fiep Bulletin 2013; ARTICLE I- 83.

15. Moura IH, Costa JV, Leal LB, Araújo DS, Silva ARV, Almeida C. Índice de massa corporal e circunferência abdominal entre adolescentes no interior do Piauí, Brasil. Rev Rene 2012; 13(2):253-260.

16. Santos JS, Costa COM, Nascimento Sobrinho CL, Silva MCM, Souza KEP, Melo BO. Perfil antropométrico e consumo alimentar de adolescentes de Teixeira de Freitas - Bahia. Rev Nutr 2005; 18(5):623-632.

17. Ogden CL, Flegal KM, Carroll MD, Johnson CL. Prevalence and trends in overweight among US children and adolescents, 1999-2000. JAMA 2002; 288(14):1728-32.

18. Linhares RS, Horta BS, Gigante DP, Dias-da-Costa JS, Olinto MTA. Distribuição de obesidade geral e abdominal em adultos de uma cidade no Sul do Brasil. Cad Saude Publica 2012; 28(3):438-448.

19. Tassitano RM, Tenório MCM, Hallal PC. Revisão sistemática sobre obesidade em adolescentes brasileiros. Rev Bras Cineantropom Desempenho Hum 2009; 11(4):449-456.

20. Gigante DP, Moura EC, Sardinha LMV. Prevalência de excesso de peso e obesidade e fatores associados, Brasil, 2006. Rev Saude Publica 2009; 43(Supl. 2):83-89. Silveira CDS, Urbanetto JS, Silva PC, Magnago TSBS,

21. Poli-de-Figueiredo CE. Perfil de sobrepeso e obesidade em trabalhadores de enfermagem em unidades de cuidado intensivo e emergência. Rev Cien Saúde 2013; 6(3):157-162.

22. Freitas JIF, Balikian JP, Miyashita LK, Neiva CM, Isidorio SCA. Crescimento e estado nutricional de crianças e adolescentes de Presidente Prudente, São Paulo, Brasil. Rev Bras Saúde Matern Infant 2008; 8(3):256-274.
23. Guedes DP, Miranda Neto JT, Almeida MJ, Silva AJRM. Impacto de fatores sociodemográficos e comportamentais Na prevalência de sobrepeso e obesidade de escolares. Rev Bras Cineantropom Desemp Hum 2010; 12(4):221-231.

24. Fischler C. Gastronomia e gastroanomia : sabedoria do corpo e crise biocultural da alimentação contemporânea. In: Contreras J, organizador. Alimentación y cultura: necessidades, gustos y costumbres. Barcelona: Universitat de Barcelona; 1995. 357-380.

25. Ferreira VA, Magalhães R. Obesidade no Brasil: tendências atuais. Rev Port Saúde Pública 2006; 24(2):71-81.

26. Silva KS, Nahas MV, Hoefelmann LP, Lopes AS, Oliveira ES. Associações entre atividade fisica, indice de massa corporal e comportamentos sedentários em adolescentes. Rev Bras Epidemiol 2008; 11(1):159-168.

27. Ferreira VA, Magalhães R. Obesidade entre os pobres no Brasil: a vulnerabilidade feminina. Cien Saude Colet 2011; 16(4):2279-2287.

28. Instituto de Pesquisa Economica Aplicada (IPEA). Pesquisa Nacional de Amostra por Domicilios - PNAD - 2008. Primeiras Analises: Demografia, Trabalho e Previdencia. Rio de Janeiro: IPEA; 2008.

29. Petribú MM, Tassitano RM, Nascimento WMF, Santos EMC, Cabral PC. Fatores associados ao sobrepeso e à obesidade em estudantes do ensino médio da rede pública estadual do município de Caruaru (PE). Rev Paul Pediatr 2011; 29(4):536-545.

30. Rossi CE, Vasconcelos FAG. Peso ao nascer e obesidade em crianças e adolescentes: uma revisão sistemática. Rev Bras Epidemiol 2010; 13(2):246-258.

31. Waller CE, DU S, Popkin BM. Patterns of overweight, inactivity, and snacking in chinese children. Obes Res 2003; 11(8):957-961.

32. Souza CO, Silva RCR, Assis AMO, Fiaccone RL, Pinto EJ, Moraes LTLP. Associação entre inatividade física excesso de peso em adolescentes de Salvador, Bahia Brasil. Rev Bras Epidemiol 2010; 13(3):468-475.

33. Benefice E, Garnier D, Simondon KB, Malina RM. Relationship between stunting in infancy and growth and fat distribution during adolescence in Senegalese girls. Eur J Clin Nutr 2001; 55(1):50-58.

34. Florêncio TM, Ferreira HS, Cavalcante JC, Luciano SM, Sawaya AL. Food consumed does not account for the higher prevalence of obesity among stunted adults in a very low-income population in the Northeast of Brazil (Maceió, Alagoas). Eur J Clin Nutr 2003; 57(11):14371446.

Artigo apresentado em 11/08/2015

Aprovado em 26/10/2015

Versão final apresentada 28/10/2015 